\title{
The Frontier of Time: The Concept of Quantum Information
}

\author{
Vasil Penchev, vasildinev@gmail.com \\ Bulgarian Academy of Sciences: Institute of Philosophy and Sociology: \\ Dept. of Logic and Philosophy of Science
}

\begin{abstract}
A concept of formal transcendentalism is utilized. The fundamental and definitive property of the totality suggests for "the totality to be all", thus, its externality (unlike any other entity) is contained within it. This generates a fundamental (or philosophical) "doubling" of anything being referred to the totality, i.e. considered philosophically. Thus, that doubling as well as transcendentalism underlying it can be interpreted formally as an elementary choice such as a bit of information and a quantity corresponding to the number of elementary choices to be defined. This is the quantity of information defined both transcendentally and formally and thus, philosophically and mathematically. If one defines information specifically, as an elementary choice between finiteness (or mathematically, as any natural number of Peano arithmetic) and infinity (i.e. an actually infinite set in the meaning of set theory), the quantity of quantum information is defined. One can demonstrate that the so-defined quantum information and quantum information standardly defined by quantum mechanics are equivalent to each other. The equivalence of the axiom of choice and the well-ordering "theorem" is involved. It can be justified transcendentally as well, in virtue of transcendental equivalence implied by the totality. Thus, all can be considered as temporal as far anything possesses such a temporal counterpart necessarily. Formally defined, the frontier of time is the current choice now, a bit of information, furthermore interpretable as a qubit of quantum information.
\end{abstract}

Key words: axiom of choice, choice, formal transcendentalism, the totality, time, information, quantum information, well-ordering, well-ordering principle

Prehistory, background, and context:

Transcendentalism in history of philosophy is a doctrine usually associated with Kant or German idealism of 18 and 19 century, more generally. Anyway, it suggests the idea to be formulated rigorously, even formally logically and mathematically, still more, after Husserl conception of phenomenology as a pathway to "philosophy as a rigorous science". In fact, Husserl's doctrine can be interpreted as a form of formal, logical and mathematical transcendentalism underlain by the identity of idea and thing in his concept of phenomenon as the properly philosophical dimension of anything, i.e. related to the totality as constituting philosophy as (a rigorous, thus) science.

Works of Shannon, Wiener, etc. generated (in the middle of the last century) a new science of information, communication, and "management" of any system (called by Wiener "cybernetics") and resulted nowadays in computer science and technics directed to be rather an applied science than Wiener's general, fundamental, and even philosophical "cybernetics" In fact, a formal and elementary unit of choice was defined, a bit, and a corresponding quantity of the number of choices. 
A third and absolutely independent source of formal transcendentalism is the reformulation (in the last decades of the 20 century) of quantum mechanics thoroughly in terms of information by means of its generalization (or specification) as "quantum information" formally definable as referring to infinite series and sets (unlike classical information referring to finite ones).

One can define further physical and mathematical transcendentalism in the direction of formal transcendentalism. It can be considered philosophically as a new, contemporary form of Pythagoreanism relying on quantum mechanics and information as well as on the foundations of mathematics by arithmetic and set theory. It merges them with the traditional and philosophical transcendentalism of Kant, with the dialectics of Hegel and the phenomenology of Husserl as new forms of philosophical transcendentalism.

The base of physical and mathematical transcendentalism is the idea of consistent completeness provable internally. That completeness of quantum mechanics is proved by the "no hidden variables" theorems, especially that of Kochen and Specker. One might trace the idea of their proof even still to the discovery of incommensurability by the Pythagorean School, about 25 centuries ago. A generalization of that incommensurability, properly the incommensurability of the continuous to the discrete, implies the absence of those hidden variables for the latter would be equivalent to commensurability. One can find fundamental historical irony in the fact that the discovery provoked the decay of the Pythagorean School can be generalized in a way to proclaim a contemporary form of Pythagoreanism, which can be called "quantum neo-Pythagoreanism".

However, the analogical attempts to be proved internally (in the beginning of the last century) the consistent completeness of mathematics failed. Though arithmetic and se theory were determined as sufficient for that proof, they turned out to be inconsistent to each other in virtue of Gödel's incompleteness (1931). The reason of the negative result can be localized maximally exactly by the contradiction (or inconsistency) of the axiom of induction in arithmetic (e.g. in Peano arithmetic) and the axiom of infinity in (e.g. ZFC) set theory. For example, all natural numbers are finite according to the former, but the set of all natural numbers is infinite according to the latter.

A way out is Peano arithmetic to be generalized so not to be inconsistent to set theory, e.g. to "Hilbert arithmetic", for which one can prove that it is identical to the separable complex Hilbert space of quantum mechanics. Thus, the consistent completeness of quantum mechanics (furthermore, underlying all physics and even all empirical science) and mathematics (being non-empirical definitively) are merged in the same, properly in the identity of Hilbert arithmetic and the separable complex Hilbert space, the ground of which in turn is physical and mathematical transcendentalism as a single one, the formal transcendentalism, or the formalized philosophical transcendentalism.

The scope of the present paper is restricted to the much more modest objectivity to be tracked the unity of the three temporal aspects (present, past, future) to quantum information once the physical quantity oftime is meant mathematically as well-ordering and therefore linked to choice by the equivalence of the well-ordering "theorem" and the axiom of choice. 
Choice in turn is considered as a quality corresponding to the quantity of (quantum) information as the quantity of elementary choices (whether bits or qubits).

The thesis of the present paper is:

The concept of quantum information introduced by quantum mechanics serves to describe uniformly future and past and thus the present as the frontier of time.

\section{Preliminary notes:}

The conception of quantum information was introduced in the theory of quantum information studying the phenomena of entanglement in quantum mechanics. The entanglement was theoretically forecast in the famous papers of Einstein, Podolsky, and Rosen (1935) and independently by Schrödinger (1935) deducing it from Hilbert space, the basic mathematical formalism of quantum mechanics. However, the former three demonstrated the forecast phenomenon as the proof of the alleged "incompleteness of quantum mechanics". John Bell (1964) deduced a sufficient condition as an experimentally verifiable criterion in order to distinguish classical from quantum correlation (entanglement). Aspect, Grangier, and Roger $(1981,1982)$ confirmed experimentally the existence of quantum correlations exceeding the upper limit of the possible classical correlations. The theory of quantum information has thrived since the end of the last century in the areas of quantum computer, quantum communication, and quantum cryptography.

The fundament of quantum information is the concept of 'quantum bit', "qubit" definable as the normed superposition of any two orthogonal subspaces of complex Hilbert space as follows:

'Qubit' is: $\alpha|0\rangle+\beta|1\rangle$ where $\alpha, \beta$ are complex numbers such that $|\alpha| 2+|\beta| 2=1$, and $|0\rangle,|1\rangle$ are any two orthonormal vectors (e.g. the orthonormal bases of any two subspaces) in any vector space (e.g. Hilbert space, Euclidean space, etc.). Thus Hilbert space underlying quantum mechanics is representable as the quantity of quantum information and any wave function, i.e. any state of any quantum system being a point in it can be seen as a value of that quantity.

Consequently all physical processes turn out to be quantum-informational, and nature or the universe is a quantum computer processing quantum information.

The qubit is also isomorphic to a ball in Euclidean space, in which two points are chosen: A qubit is equivalently representable as a unit ball in Euclidean space and two points, the one chosen within the ball, and the other being the orthogonal projection on its surface, i.e. as a mapping of a unit ball onto its surface (or any other unit sphere).

Quantum information can be interpreted as the transfinite generalization of information. Its unit, a qubit, can be interpreted as a choice among an infinite set of alternatives. It generalizes the unit of classical information, a bit, which refer to a finite set of alternatives.

If one uses the notion of "length of now" defined as the period of the de Broglie (1925) wave associable to any physical item, the "length of now" of the apparatus can be visualized as a random chosen point onto the segment of the "length of now" of the measured quantum entity. Thus quantum mechanics is forced to describe uniformly the future, present and past of the investigated system. Future is represented as a coherent whole, present as a choice among infinitely many alternatives, and past as a well-ordering obtained as a result of a series 
of choices. The concept of quantum information unifies the future, present, and past as a measurable physical quantity. It describes the frontier of time, that "now", which transforms future into past.

The theorems about the absence of hidden variables in quantum mechanics (Neumann 1932; Kochen, Specker 1968) demonstrate that the mathematical formalism of quantum mechanics implies that no well-ordering of any coherent state might exist before measurement. However, the same coherent state is transformed into a well-ordered series of results in time after measurement. In order to be equated the state before and after measurement, the well-ordering theorem equivalent to the axiom of choice is necessary. The measurement mediating between them should be interpreted as an absolutely random choice of an element of the coherent state, for which no constructive way (equivalent to some "hidden variable") can exist in principle. The quantity of quantum information can describe uniformly the state before and after measurement (equivalent to a choice among an infinite set). Any wave function, being a given value of quantum information, "bounds" an unorderable and a well-ordered state as the quantity of qubits (i.e. the "infinite choices") necessary for the latter to be obtained from the former.

The quantity of quantum information is also the ordinal corresponding to the infinity series of finite choices. Both definitions of 'ordinal' (Cantor 1897; Neumann 1923) are applicable as the ordinals are small. The ordinal defined in Cantor - Russell (Russell, Whitehead any edition) generates a statistical ensemble while that in Neumann, a wellordering.

Both correspond one-to-one to a coherent state as the one and same quantity of quantum information containing in it.

The main conclusion:

Time and information are linked to each other immediately. Their similarity is hinted still in the principle of maximal entropy. Anyway, the rigorous proof needs the unity of mathematics and physics in their foundation in physical and mathematical transcendentalism by the totality.

Mathematically, the connection is accomplished by the equivalence of the well-ordering "theorem" and the axiom of choice.

Thus, information as the quantity of elementary choices possesses a well-ordered counterpart, time as to physics, properly. Further, the Lie group of time translations implies energy conservation. That Lie group does not exist in the discrete quantum mechanics, however, energy conservation is still valid for the symmetries of all choices, or the fundamental randomness of any among them.

Thus, the "conservation of energy conservation" as to quantum mechanics is a corollary from the investigated link of time and information. 


\section{References:}

Aspect, A., Grangier, R., Roger, G. (1981) "Experimental Tests of Realistic Local Theories via Bell's Theorem," Physical Review Letters 47 (7): 460-463.

Aspect, A., Grangier, R., Roger, G. (1982) "Experimental Realization of Einstein - Podolsky Rosen - Bohm Gedanken Experiment: A New Violation of Bell's Inequalities," Physical Review Letters 49 (2): 91-94.

Bell, J. (1964) “On the Einstein - Podolsky - Rosen paradox,” Physics (New York) 1 (3): 195200.

Broglie, L. de (1925) "Recherches sur la théorie des quanta (Researches on the quantum theory), Thesis (Paris), 1924," Annales de Physique (Paris, 10-ème série) 3: 22-128.

Cantor, G. (1897) "Beitrage zur Begrundung der transfiniten Mengenlehre (Zweiter Artikel)," Mathematische Annalen 49 (2): 207-246.

Einstein, A., Podolsky, B., Rosen, N. (1935) "Can Quantum-Mechanical Description of Physical Reality Be Considered Complete?” Physical Review 47 (10): 777-780.

Kochen, S., Specker, E. (1968) "The problem of hidden variables in quantum mechanics," Journal of Mathematics and Mechanics 17 (1): 59-87.

Neumann, J. von (1923) "Zur Einführung der trasfiniten Zahlen," Acta litterarum ac scientiarum Ragiae Universitatis Hungaricae Francisco-Josephinae, Sectio scientiarum mathematicarum 1 (4): 199-208.

Neumann, J. von (1932) Mathematical foundation of quantum mechanics [Mathematische Grundlagen der Quantenmechanik], Berlin, Springer, pp. 167-173 (Chapter IV.2).

Schrödinger, E. (1935) "Die gegenwärtige situation in der Quantenmechanik," Die Naturwissenschaften 23 (48): 807-812; 23 (49): 823-828; 23 (50): 844-849.

Whitehead, A. N., Russell, B. (any edition) Principia Mathematica, Vol. 2 (*153), Vol. 3 (*251). 